\title{
Reducing radicals in the spirit of Euclid*
}

\author{
Kurt Girstmair
}

\begin{abstract}
Let $p$ be an odd natural number $\geq 3$. Inspired by results from Euclid's Elements, we express the irrational

$$
y=\sqrt[p]{d+\sqrt{R}}
$$

whose degree is $2 p$, as a polynomial function of irrationals of degrees $\leq p$. In certain cases $y$ is expressed by simple radicals. This reduction of the degree exhibits remarkably regular patterns of the polynomials involved. The proof is based on hypergeometric summation, in particular, on Zeilberger's algorithm.
\end{abstract}

\section{Introduction and main result}

In the tenth book of Euclid's elements, Proposition 54, one finds an answer to the following question: Suppose that the biquadratic radical

$$
y=\sqrt{d+\sqrt{R}}
$$

is given, where $d$ and $R$ are positive rational numbers, $\sqrt{R} \notin \mathbb{Q}$ and $y \notin \mathbb{Q}(\sqrt{R})$. When is it possible to express $y$ in terms of two simple square roots? Euclid's answer is as follows: If $d^{2}-R$ is a square, i.e., $d^{2}-R=k^{2}, k \in \mathbb{Q}$, then

$$
y=\sqrt{\frac{d+k}{2}}+\sqrt{\frac{d-k}{2}} .
$$

But, to tell the truth, Euclid has no formulas, and his answer is phrased in purely geometric terms. So (11) is a modern algebraic interpretation of what Euclid did in the framework of his geometry (see [3, p. 119]).

Formula (1) has been considered as an example of denesting a nested radical (see [1], Th. 1). Our viewpoint, however, is different, as we illustrate with the example

$$
y=\sqrt[4]{d+\sqrt{R}}
$$

Here we assume that $y$ is an irrational of degree 8 , which means that the polynomial $\left(Z^{4}-d\right)^{2}-R$ is irreducible. Provided that $d^{2}-R=k^{4}, k \in \mathbb{Q}$, we can apply Euclid's result twice and obtain

$$
y=\sqrt{\sqrt{\frac{d+k^{2}}{8}}+\frac{k}{2}}+\sqrt{\sqrt{\frac{d+k^{2}}{8}}-\frac{k}{2}} .
$$

*2010 MSC: 12F10; 33F10. Keywords: Radical extensions; Zeilberger's algorithm. 
So (3) does not denest the nested radical (2). Nevertheless, this identity can be considered as a reduction of the degree, inasmuch as it expresses an irrational of degree 8 as a sum of two irrationals of degree 4 .

The present paper is devoted to this kind of reduction in the case of the radical

$$
y=\sqrt[p]{d+\sqrt{R}}
$$

where $p \geq 3$ is an odd natural number. To this end let $K$ be a field of characteristic 0 and let $d, R \in K \backslash\{0\}$. By $\bar{K}$ we denote an algebraic closure of $K$. An irrational is an element of $\bar{K} \backslash K$. The degree of an irrational is the degree of its minimal polynomial over $K$. Let $\sqrt{R}$ be an irrational. More precisely, we select one of the two possible values of $\sqrt{R}$, whereas the other value is denoted by $-\sqrt{R}$. This meaning of $\sqrt{R}$ shall be fixed throughout. Suppose that $y$ is an irrational of degree $2 p$. This is the same as saying that the polynomial

$$
g=\left(Z^{p}-d\right)^{2}-R
$$

is irreducible in the polynomial ring $K[Z]$. We are going to reduce $y$ to two irrationals of degree $\leq p$ together with $\sqrt{R}$, i.e., we express $y$ as a polynomial function (with coefficients in $K$ ) of these quantities. Surprisingly, this can be done by means of explicit formulas valid for all odd natural numbers $p$.

For this purpose we work with the decomposition

$$
g=h \cdot h^{\prime} \quad \text { with } \quad h=Z^{p}-d-\sqrt{R}, \quad h^{\prime}=Z^{p}-d+\sqrt{R},
$$

which takes place in the polynomial ring $K(\sqrt{R})[Z]$. Now suppose that $y$ is a zero of $h$, whereas $y^{\prime}(\in \bar{K})$ is a zero of $h^{\prime}$. We put

$$
z=y y^{\prime} \text { and } u=z^{(p-1) / 2}\left(y+y^{\prime}\right) .
$$

Then $z$ is a $p$ th root of

$$
D=d^{2}-R
$$

i.e., $z^{p}=D \in K$. By our assumptions, $D \neq 0$. On the other hand, we will see that $u$ is a zero of the polynomial

$$
f=D^{(p-1) / 2} \sum_{k=0}^{(p-1) / 2} \frac{c_{2 k+1}}{D^{k}} Z^{2 k+1}-2 d D^{(p-1) / 2} \in K[Z],
$$

where $D$ is defined by (66) and $c_{2 k+1}$ by

$$
c_{2 k+1}=(-1)^{(p-1) / 2-k} \frac{p}{\frac{p+1}{2}+k}\left(\begin{array}{c}
\frac{p+1}{2}+k \\
2 k+1
\end{array}\right),
$$

$k=0, \ldots,(p-1) / 2$. Here $\left(\begin{array}{c}m \\ n\end{array}\right)$ is the usual binomial coefficient. Note that $f$ is a polynomial of degree $p$ with leading coefficient $c_{p}=1$. Define the polynomial $A \in K[Z]$ by

$$
A=\frac{1}{2 R} \sum_{k=0}^{(p-1) / 2} \frac{a_{2 k}}{D^{k}} Z^{2 k}+(-1)^{(p+1) / 2} \frac{d}{2 R D} Z
$$


with

$$
a_{2 k}=(-1)^{k} \frac{p-1}{\frac{p-1}{2}+k}\left(\begin{array}{c}
\frac{p-1}{2}+k \\
2 k
\end{array}\right),
$$

$k=0, \ldots,(p-1) / 2$. Then our main result reads as follows.

Theorem 1 Let $y, y^{\prime}, z=y y^{\prime}$, and $u=z^{(p-1) / 2}\left(y+y^{\prime}\right)$ be as above. In particular, $\sqrt{R} \notin K, y, y^{\prime}$ are irrationals of degree $2 p$, and $u$ is a zero of $f$. Then

$$
\left\{y, y^{\prime}\right\}=\left\{z^{(p+1) / 2}\left(\frac{u}{2 D} \pm A(u) \sqrt{R}\right)\right\}
$$

Remarks. 1. In our setting, $z$ is a $p$ th root of $D$ and $u$ a zero of the polynomial $f$ of degree $p$ in $K[Z]$. Therefore, (11) reduces the radical $y$ of (44), whose degree is $2 p$, to $z$, $u$ and $\sqrt{R}$, whose degrees are $\leq p$ and $=2$, respectively.

2. Instead of choosing $y, y^{\prime}$ in the above way, one may choose a zero $y$ of $h$ and a $p$ th root $z$ of $D$. Then $y^{\prime}=z / y$ is a zero of the polynomial $h^{\prime}$.

3. We will see below (Proposition 1) that for a given $p$ th root $z$ of $D$ and a given zero $u$ of $f$ there is exactly one zero $y$ of h such that for $y^{\prime}=z / y$ we have $u=z^{(p-1) / 2}\left(y+y^{\prime}\right)$.

The proof of Theorem 1 is based on techniques of hypergeometric summation, in particular, on Zeilberger's algorithm. We think that one can hardly dispense with these techniques or, in other words, a proof without algorithmic methods of this kind seems to be out of reach.

Example 1. We consider the special case $p=5$. Here we have

$$
f=Z^{5}-5 D Z^{3}+5 D^{2} Z-2 d D^{2} \text { and } A=\frac{1}{R}\left(\frac{Z^{4}}{2 D}-\frac{2 Z^{2}}{D}-\frac{d Z}{2 D}+1\right) .
$$

In this case the polynomial $f$ is called DeMoivre's quintic (see [2], [1]). If, for instance $d=2$ and $R=5$, we obtain $g=Z^{10}-4 Z^{5}-1$, which is irreducible over $K=\mathbb{Q}$. Moreover, $D=-1$, and we can choose $z=-1$ (in the sense of Remark 2). Finally, $f=Z^{5}+5 Z^{3}+5 Z-4$ is irreducible over $\mathbb{Q}$ and $A=\left(Z^{4}+4 Z^{2}+2 Z+2\right) / 10$.

Let $z$ be a $p$ th root of $D$ and suppose that $f$ has a zero $u$ in the ground field $K$. By Remark 3, there is a uniquely determined zero $y$ of $h$ such that $u=z^{(p-1) / 2}\left(y+y^{\prime}\right)$ for $y^{\prime}=z / y$. Then (11) expresses $y$ and $y^{\prime}$ as polynomial functions of $z$ and $\sqrt{R}$ with coefficients in $K$. In other words, the nested radicals $y$ and $y^{\prime}$ are denested in this way.

Example 2. Let $K=\mathbb{Q}$ and $p=7$. Put $d=-2158$ and $R=6 \cdot 881^{2}$. Thus, $d+\sqrt{R}=$ $-2158+881 \sqrt{6}$ and $D=-2$. In this case the polynomial $g$ equals $Z^{14}+4316 Z^{7}-2$ and is irreducible. It turns out that $u=4$ is a zero of $f$. We choose $z=-2^{1 / 7}$, where $2^{1 / 7}$ is the real 7 th root of 2 . If the zero $y$ of $h$ is determined by $u=z^{(p-1) / 2}\left(y+y^{\prime}\right), y^{\prime}=z / y$, then formula (11) gives

$$
\left\{y, y^{\prime}\right\}=\left\{2^{4 / 7}(-1 \pm \sqrt{6} / 2)\right\}
$$

The problem of denesting radicals has attracted considerable attention, see, for instance, [12], [5], 8]. Further references can be found in [9].

Sections $2-4$ are devoted to the proof of Theorem 1 . 
Section 5 contains the aforementioned Proposition 1. Moreover, we show how to find examples like the above Example 2 in a simple way.

In Section 6 we discuss the question whether $f$ can be replaced by a polynomial of the form $Z^{p}-a, a \in K$, which is the same as replacing $u$ by the $p$ th root of an element of $K$ (Proposition 2). In this case our reduction could be replaced by a reduction which also denests the radical $y$. In addition, we consider two cases where (111) is equivalent to expressing $y, y^{\prime}$ in terms of a $\mathbb{Q}$-basis of the field $\mathbb{Q}(z, u, \sqrt{R})$ (Proposition 3). These cases seem to be generic, inasmuch as most examples fall under them. We also get some insight into the structure of the splitting field of the polynomial $g$.

\section{The polynomial $f$}

We adopt the above notation. In particular, $f$ is defined by (7) and (86). We have to show that $u=z^{(p-1) / 2}\left(y+y^{\prime}\right)$ is a zero of $f$.

For this purpose we look at the following expansion of the polynomial $X^{p}+1 \in \mathbb{Q}[X]$ :

$$
X^{p}+1=\sum_{k=0}^{(p-1) / 2} C_{p-2 k} X^{k}(X+1)^{p-2 k}
$$

with rational coefficients $C_{p-2 k}$. We will see that this expansion exists and is unique. Indeed, if we apply the binomial formula to $(X+1)^{p-2 k}$ and consider only the coefficients of the monomials $X^{j}, j=0, \ldots,(p-1) / 2$, we obtain

$$
\sum_{j=0}^{(p-1) / 2} X^{j} \sum_{k=0}^{j} C_{p-2 k}\left(\begin{array}{c}
p-2 k \\
j-k
\end{array}\right)=1
$$

This gives the system of linear equations

$$
\begin{aligned}
& 1=C_{p}, \\
& 0=C_{p}\left(\begin{array}{l}
p \\
1
\end{array}\right)+C_{p-2}\left(\begin{array}{c}
p-2 \\
0
\end{array}\right) \text {, } \\
& 0=C_{p}\left(\begin{array}{l}
p \\
2
\end{array}\right)+C_{p-2}\left(\begin{array}{c}
p-2 \\
1
\end{array}\right)+C_{p-4}\left(\begin{array}{c}
p-4 \\
0
\end{array}\right) \text {, } \\
& 0=\sum_{k=0}^{j} C_{p-2 k}\left(\begin{array}{c}
p-2 k \\
j-k
\end{array}\right) .
\end{aligned}
$$

One immediately sees that the coefficients $C_{p-2 k}, k=0, \ldots, p-1$, are uniquely determined by (13).

Let $F$ denote the polynomial on the right hand side of (12). We obtain

$$
F\left(y / y^{\prime}\right) \cdot y^{\prime p}=\sum_{k=0}^{(p-1) / 2} C_{p-2 k} z^{k}\left(y+y^{\prime}\right)^{p-2 k}=y^{p}+y^{\prime p}=2 d
$$


since $y^{p}=d+\sqrt{R}, y^{p}=d-\sqrt{R}$ (see (5) ). If we multiply the identity (14) by $D^{(p-1) / 2}=$ $z^{p(p-1) / 2}$, we have

$$
2 d D^{(p-1 / 2)}=\sum_{k=0}^{(p-1) / 2} C_{p-2 k} D^{k} z^{((p-1) / 2) \cdot(p-2 k)}\left(y+y^{\prime}\right)^{p-2 k} .
$$

In other words, $u=z^{(p-1) / 2}\left(y+y^{\prime}\right)$ is a zero of $f$, provided that the coefficients $C_{p-2 k}$ of (12) coincide with the coefficients $c_{p-2 k}$ of $f, k=0, \ldots,(p-1) / 2$.

We will show that the coefficients $c_{p-2 k}$ satisfy the system (13) of linear equations. By (8),

$$
c_{p-2 k}=(-1)^{k} \frac{p}{p-k}\left(\begin{array}{c}
p-k \\
k
\end{array}\right)
$$

$k=0, \ldots,(p-1) / 2$. We recall that $c_{p}=1$ and observe that the right hand side of (13) reads, for these values of $c_{p-2 k}$,

$$
\sum_{k=0}^{j}(-1)^{k} \frac{p}{p-k}\left(\begin{array}{c}
p-k \\
k
\end{array}\right)\left(\begin{array}{c}
p-2 k \\
j-k
\end{array}\right)
$$

$j=1, \ldots,(p-1) / 2$. This is a typical example of a hypergeometric summation (see [7, chap. 2]). The summand of (15) is defined for all integers $k \in \mathbb{Z}$, since $\left(\begin{array}{c}p-k \\ k\end{array}\right) /(p-k)=$ $\left(\begin{array}{c}p-k-1 \\ k-1\end{array}\right) / k$ for all $k \neq 0$. In particular, it takes the value 0 for all $k>j$ and all $k<0$. For our purpose it is advisable to change the summation order, i.e., we consider

$$
\sum_{k=0}^{j}(-1)^{j-k} \frac{p}{p-j+k}\left(\begin{array}{c}
p-j+k \\
j-k
\end{array}\right)\left(\begin{array}{c}
p-2 j+2 k \\
k
\end{array}\right) .
$$

Let $b_{k}$ denote the summand of (16). Hypergeometric summation requires considering $b_{k+1} / b_{k}, k=0, \ldots, j$, and $b_{0}$. We have

$$
\frac{b_{k+1}}{b_{k}}=\frac{(k-j)(k+p-j)}{(k+p-2 j+1)(k+1)} .
$$

Since $b_{0}=(-1)^{j} \frac{p}{p-j}\left(\begin{array}{c}p-j \\ j\end{array}\right)$, we obtain that (15) equals

$$
(-1)^{j} \frac{p}{p-j}\left(\begin{array}{c}
p-j \\
j
\end{array}\right){ }_{2} F_{1}\left(\begin{array}{c}
-j, p-j \\
p-2 j+1
\end{array} \mid 1\right)
$$

$j=1, \ldots,(p-1) / 2$, where ${ }_{2} F_{1}$ denotes Gauss' hypergeometric function. A theorem of Gauss (see [7, p. 32]) says

$$
{ }_{2} F_{1}\left(\begin{array}{c|c}
-j, b & 1 \\
c & 1
\end{array}\right)=\frac{(c-b)_{j}}{(c)_{j}}
$$

here $(a)_{j}$ is given by $(a)_{j}=a \cdot(a+1) \cdots(a+j-1)$ and $b, c \in \mathbb{Z}, c>0$. In our case, $c=p-2 j+1>0$ and, thus, $(c)_{j} \neq 0$. On the other hand, $c-b=-j+1$, which means $(c-b)_{j}=0$. Hence the sum (15) vanishes for all $j=1, \ldots,(p-1) / 2$.

We remark that the coefficients of the monomials $X^{j}, j=(p+1) / 2, \ldots, p$, in (12) also yield the system (13) for the numbers $C_{p-2 k}$. 


\section{The basic identity}

We return to Theorem 1. Indeed, (11) is the same as saying

$$
\pm z^{(p+1) / 2} A(u) \sqrt{R}=\frac{y-y^{\prime}}{2} .
$$

For instance, the plus-sign on the left hand side of (17) gives

$$
y=\frac{y+y^{\prime}}{2}+\frac{y-y^{\prime}}{2}=\frac{u}{2 z^{(p-1) / 2}}+z^{(p+1) / 2} A(u) \sqrt{R},
$$

which obviously has the form of (11). However, (17) is equivalent to

$$
z^{p+1} A(u)^{2} R=\frac{\left(y+y^{\prime}\right)^{2}-4 y y^{\prime}}{4} .
$$

Since the right hand side of this identity equals $\left(u^{2}-4 z^{p}\right) /\left(4 z^{p-1}\right)$, we see that (17) is equivalent to

$$
4 D^{2} A(u)^{2} R=u^{2}-4 D
$$

We define

$$
f^{\prime}=\frac{1}{R D^{(p-3) / 2}} \sum_{j=0}^{(p-3) / 2} \frac{c_{2 j+1}^{\prime}}{D^{j}} Z^{2 j+1}-\frac{2 d}{R D^{(p-3) / 2}}
$$

with

$$
c_{2 j+1}^{\prime}=(-1)^{(p-3) / 2-j} \frac{p-2}{\frac{p-1}{2}+j}\left(\begin{array}{c}
\frac{p-1}{2}+j \\
2 j+1
\end{array}\right) .
$$

We are going to prove the identity of polynomials

$$
4 D^{2} A^{2} R=f \cdot f^{\prime}+Z^{2}-4 D .
$$

If we insert $u$ for the variable $Z$ in (19) and observe $f(u)=0$, we obtain (18). Hence (19) can be considered as the fundamental identity of this paper. The proof of this identity consists in comparing the coefficients of the monomials $Z^{m}$ on both sides.

The following three cases have to be distinguished. First, $m \in\{0,2\}$, second, $m$ odd, and third, $m=2 k, k=2, \ldots, p-1$. The case $m \in\{0,2\}$ may be checked by the reader. In the remaining cases, we write $\alpha_{m}$ for the coefficient of $Z^{m}$ on the left hand side of (19) and $\beta_{m}$ for the coefficient of $Z^{m}$ on the right hand side. In view of (9), (10), we obtain

$$
R D^{k-1} \alpha_{2 k+1}=(-1)^{\frac{p+1}{2}+k} 2 d \cdot \frac{p-1}{\frac{p-1}{2}+k}\left(\begin{array}{c}
\frac{p-1}{2}+k \\
2 k
\end{array}\right)
$$

and

$$
R D^{k-1} \beta_{2 k+1}=(-1)^{\frac{p+1}{2}+k} 2 d \cdot\left(\frac{p}{\frac{p+1}{2}+k}\left(\begin{array}{c}
\frac{p+1}{2}+k \\
2 k+1
\end{array}\right)-\frac{p-2}{\frac{p-1}{2}+k}\left(\begin{array}{c}
\frac{p-1}{2}+k \\
2 k+1
\end{array}\right)\right)
$$

$k=0, \ldots,(p-1) / 2$ (observe that $\left.\left(\begin{array}{c}p-1 \\ p\end{array}\right)=0\right)$. Using elementary identities of binomial coefficients, one sees that $\alpha_{2 k+1}=\beta_{2 k+1}$. 
The remaining case is the most difficult one. We obtain

$$
R D^{k-2} \alpha_{2 k}=(-1)^{k} \sum_{j=0}^{k} \frac{(p-1)^{2}}{\left(\frac{p-1}{2}+j\right)\left(\frac{p-1}{2}+k-j\right)}\left(\begin{array}{c}
\frac{p-1}{2}+j \\
2 j
\end{array}\right)\left(\begin{array}{c}
\frac{p-1}{2}+k-j \\
2(k-j)
\end{array}\right)
$$

and

$$
R D^{k-2} \beta_{2 k}=(-1)^{k} \sum_{j=0}^{k-1} \frac{p(p-2)}{\left(\frac{p+1}{2}+j\right)\left(\frac{p-1}{2}+k-j-1\right)}\left(\begin{array}{c}
\frac{p+1}{2}+j \\
2 j+1
\end{array}\right)\left(\begin{array}{c}
\frac{p-1}{2}+k-j-1 \\
2(k-j-1)+1
\end{array}\right)
$$

for $k=2, \ldots,(p-1)$. Here we observe that the left binomial coefficient in (20) as well as in (21) equals 0 if $j>(p-1) / 2$. In the same way the right binomial coefficient vanishes in both identities if $k-j>(p-1) / 2$. We also observe that the sum on the right hand side of (21) may be extended to the upper bound $k$ (instead of $k-1$ ) since the respective summand is 0 . In the next section we show that $\alpha_{2 k}=\beta_{2 k}, k=2, \ldots, p-1$.

\section{Two hypergeometric summations}

In this section we denote the right hand side of (20) by $s_{k}$ and the right hand side of (21) by $t_{k}$ for $k=0, \ldots, p-1$. Further, we introduce

$$
u_{k}=\frac{(-1)^{k}(p-1)}{k}\left(\begin{array}{c}
p+k-2 \\
2 k-1
\end{array}\right)
$$

$k=1, \ldots, p-1$. We will show that $s_{k}=u_{k}$ and $t_{k}=u_{k}$ for all $k=2, \ldots, p-1$. In this way we also exhibit the value of the coefficient $\alpha_{2 k}$ of $4 D^{2} A^{2} R$.

Zeilberger's algorithm yields recursion formulas for $s_{k}$ and $t_{k}$ (see [7, chap. 7]). In the case of $s_{k}$, this formula reads

$$
\left(4 k^{2}+6 k+2\right) s_{k+1}+\left(-k^{2}-2 p+1+p^{2}\right) s_{k}=0 .
$$

Note that $4 k^{2}+6 k+2 \neq 0$ for $k \geq 0$. Now $u_{k} \neq 0$ for $k=1, \ldots, p-2$ and

$$
\frac{u_{k+1}}{u_{k}}=-\frac{-k^{2}-2 p+1+p^{2}}{4 k^{2}+6 k+2} \text {. }
$$

In particular, $u_{k}$ satisfies formula (22) . Moreover, $u_{1}=s_{1}=-(p-1)^{2}$. Hence $u_{k}=s_{k}$ for all $k=1, \ldots, p-1$.

In the case of $t_{k}$ Zeilberger's algorithm yields

$$
a \cdot t_{k+2}+b \cdot t_{k+1}+c \cdot t_{k}=0
$$

with

$$
\begin{aligned}
a & =16 k^{3}+64 k^{2}+76 k+24, b=-8 k^{3}-12 k^{2}-8 p k+4 p^{2} k+2 p^{2}-4 p+2, \\
c & =k^{3}-k^{2}-p^{2} k+2 p k-k+p^{2}-2 p+1 .
\end{aligned}
$$

Again, $a \neq 0$ for all $k \geq 0$. It is not hard to check that

$$
a \cdot \frac{u_{k+2}}{u_{k}}+b \cdot \frac{u_{k+1}}{u_{k}}+c=0
$$

for $k=2, \ldots, p-3$. Since $t_{2}=u_{2}=p(p-1)^{2}(p-2) / 12$ and $t_{3}=u_{3}=-p(p-1)^{2}(p-$ $2)(p-3)(p+1) / 360$, we obtain $u_{k}=t_{k}$ for all $k=2, \ldots, p-1$. 


\section{Some additional observations}

Let the above assumptions hold, in particular, $d \neq 0$ and $\sqrt{R} \notin K$.

Proposition 1 Let $z$ be an arbitrary pth root of $D$ and $u$ a zero of $f$. Then there is a uniquely determined zero $y$ of $h$ such that for $y^{\prime}=z / y$ we have $u=z^{(p-1) / 2}\left(y+y^{\prime}\right)$.

Proof. Let $V(h)$ and $V(f)$ denote the sets of the zeros (in $\bar{K}$ ) of $h$ and $f$, respectively. Then the map

$$
V(h) \rightarrow V(f): y \mapsto z^{(p-1) / 2}(y+z / y)
$$

is well defined (recall Remark 2 in Section 1). This map is injective. Indeed, if $u \in V(f)$ equals $z^{(p-1) / 2}(y+z / y)$ for some $y \in V(h)$, then the quadratic equation

$$
z^{(p-1) / 2}(x+z / x)=u
$$

has at most two solutions, namely, $x=y$ and $x=z / y$. However, $z / y$ is a zero of $h^{\prime}$, and $h$ and $h^{\prime}$ have no common zero since $\sqrt{R} \neq 0$. Hence $y$ is the only solution of this equation in $V(h)$.

Since $h$ has no multiple zeros, the set $V(h)$ has $p$ elements and $V(f)$ at most $p$. By the injectivity, $V(f)$ also has $p$ elements and the map of (23) is bijective. This proves our assertion.

Our next aim is a simple construction of examples like Example 2 in Section 1. Suppose that $D \in K \backslash\{0\}$ and $u \in K$ are given. Then the equation $f(u)=0$ holds if, and only if,

$$
d=\frac{1}{2} \sum_{j=0}^{(p-1) / 2} \frac{c_{2 j+1}}{D^{j}} u^{2 j+1}
$$

(recall (7), (8)). For instance, if $D=-2$ and $u=4$, we obtain $d=-2158$ (see the aforementioned example). Then we determine $R$ by $R=d^{2}-D$. In the case $K=\mathbb{Q}$ it frequently happens that $\sqrt{R} \notin \mathbb{Q}$ and that $g=\left(Z^{p}-d\right)^{2}-R$ is irreducible in $\mathbb{Q}[Z]$. Choose a $p$ th root $z$ of $D($ in $\bar{K}))$. Then the zero $y$ of $h$ is uniquely determined as a solution of the quadratic equation (24); and $y$ and $y^{\prime}=z / y$ can be obtained by (11). In general, however, it is simpler to obtain $y, y^{\prime}$ as solutions of the quadratic equation provided that $u$ is known. In this case

$$
\left\{y, y^{\prime}\right\}=\left\{\frac{1}{2 z^{(p-1) / 2}}\left(u \pm \sqrt{u^{2}-4 z^{p}}\right)\right\} .
$$

Since $u$ and $z^{p}=D \in K$, we have $u^{2}-4 z^{p} \in K$. Of course, (25) is equivalent to (11) in this context.

\section{Two further results}

The question arises whether the zero $u$ of $f$ can be replaced by the $p$ th root of an element of $K$. This would imply that the splitting field $L$ of $f$ is also the splitting field of a polynomial $P$ of the form $P=Z^{p}-a$ for some $a \in K$. 
In order to obtain a partial answer, we suppose that $p \geq 3$ is a prime and $K=\mathbb{Q}$. Further, we assume that $f$ is irreducible over $\mathbb{Q}$. If $L$ is also the splitting field of $P$, then $P$ is irreducible over $\mathbb{Q}$ and $L$ contains a primitive $p$ th root of unity $\zeta_{p}$. Indeed, $L=\mathbb{Q}\left(v, \zeta_{p}\right)$, where $v$ is a zero of $P$.

Proposition 2 As above, let $K=\mathbb{Q}, p \geq 3$ a prime and $f$ irreducible in $\mathbb{Q}[Z]$. If $\mathbb{Q}(\sqrt{R}) \neq \mathbb{Q}\left(\sqrt{(-1)^{(p-1) / 2} p}\right)$, then $\zeta_{p} \notin L$. In particular, $L$ is not the splitting field of a polynomial $P$ as above.

Proof. Let $y$ be a zero of $h, y^{\prime}$ a zero of $h^{\prime}$, and $z=y y^{\prime}$. The bijection (23) implies that the numbers $u_{k}=z^{(p-1) / 2}\left(y \zeta_{p}^{k}+y^{\prime} \zeta_{p}^{-k}\right), k=0, \ldots, p-1$, are exactly the zeros of $f$. The Lagrange resolvent

$$
\sum_{k=0}^{p-1} \zeta_{p}^{-k} u_{k}=z^{(p-1) / 2} y p+z^{(p-1) / 2} y^{\prime} \sum_{k=0}^{p-1} \zeta_{p}^{-2 k}=z^{(p-1) / 2} y p
$$

shows $z^{(p-1) / 2} y \in L\left(\zeta_{p}\right)$ and, thus, $D^{(p-1) / 2} y^{p}=D^{(p-1) / 2}(d+\sqrt{R}) \in L\left(\zeta_{p}\right)$. In particular, $\sqrt{R} \in L\left(\zeta_{p}\right)$. Observe that all elements of $L$ of a degree different from $p$ are contained in a uniquely determined subfield $L_{1}$, whose degree (over $\mathbb{Q}$ ) divides $p-1$ (see [4, p. 163]). Suppose that $\zeta_{p} \in L$. Then $\zeta_{p} \in L_{1}$, and, therefore, $L_{1}=\mathbb{Q}\left(\zeta_{p}\right)$ since $\zeta_{p}$ has the degree $p-1$ over $\mathbb{Q}$. Moreover, $\sqrt{R} \in L_{1}$. The field $\mathbb{Q}\left(\zeta_{p}\right)$ has a cyclic Galois group of order $p-1$ over $\mathbb{Q}$. Accordingly, it contains a uniquely determined quadratic subfield, namely, $\mathbb{Q}\left(\sqrt{(-1)^{(p-1) / 2} p}\right)$ (see [6, p. 71$\left.]\right)$. This implies $\mathbb{Q}(\sqrt{R})=\mathbb{Q}\left(\sqrt{(-1)^{(p-1) / 2} p}\right)$.

Next we investigate the connection between formula (11) and $\mathbb{Q}$-bases.

Proposition 3 As above, let $K=\mathbb{Q}, p \geq 3$ a prime and $f$ irreducible in $\mathbb{Q}[Z]$. In addition, suppose that $\zeta_{p}$ is not contained in the splitting field of $f$.

(a) If $D=z^{p}$ for some $z \in \mathbb{Q}$, then $u^{k} \sqrt{R}^{l}, k=0, \ldots, p-1, l=0,1$, is a $\mathbb{Q}$-basis of $\mathbb{Q}(y)=\mathbb{Q}\left(y^{\prime}\right)=\mathbb{Q}(u, \sqrt{R})$.

(b) Suppose that $D$ does not have this form. Let $z$ be a pth root of $D$. Then $z^{j} u^{k} \sqrt{R}^{l}$, $j, k=0, \ldots, p-1, l=0,1$, is a $\mathbb{Q}$-basis of $\mathbb{Q}(z, u, \sqrt{R})$.

In both cases, (11) expresses $y, y^{\prime}$ in terms of the respective basis.

Proof. Assertion (a) is obvious. Suppose that $D$ does not have the form of (a). Then $Z^{p}-D$ is irreducible over $\mathbb{Q}$ (see $[10$, p. 221]). Let $z$ be a $p$ th root of $D$. Suppose that $Z^{p}-D$ has a zero in $\mathbb{Q}(u)$. This means that $z \zeta_{p}^{k} \in \mathbb{Q}(u)$ for some $k \in\{0, \ldots, p-1\}$. But then $\mathbb{Q}(u)=\mathbb{Q}\left(z \zeta_{p}^{k}\right)$, because both $f$ and $Z^{p}-D$ are irreducible. Since the splitting field $L$ of $f$ is a Galois extension of $\mathbb{Q}$, this implies that $Z^{p}-D$ splits into linear factors over $L$. In particular, $\zeta_{p} \in L$, which we have excluded. Hence $Z^{p}-D$ has no zero in $\mathbb{Q}(u)$, and, by the cited argument, $Z^{p}-D$ is irreducible over $\mathbb{Q}(u)$. Accordingly, $\mathbb{Q}(z, u)$ has the degree $p^{2}$ over $\mathbb{Q}$. This field does not contain $\sqrt{R}$ for reasons of degree. Altogether, the field $\mathbb{Q}(z, u, \sqrt{R})$ has the degree $2 p^{2}$ over $\mathbb{Q}$ and the $\mathbb{Q}$-basis of (b). Now it is clear that (11) expresses $y, y^{\prime}$ in terms of this basis.

Note that in case (b) neither $y \in \mathbb{Q}(u, \sqrt{R})$ nor $y \in \mathbb{Q}(z, \sqrt{R})$. Observe, further, that in this case only $(p+1) / 2+2$ of a total of $2 p^{2}$ basis vectors actually occur in (11). It 
seems that Propositions 2 and 3 cover the generic case, i.e., most examples satisfy the assumptions of these propositions.

Let us briefly look at the splitting field $M$ of the polynomial $g$ in case (b). If $L$ denotes the splitting field of $f$, then $M$ is the composite of the Galois extensions $\mathbb{Q}\left(z, \zeta_{p}\right)$ and $L$ of $\mathbb{Q}$ (recall that $\sqrt{R} \in L\left(\zeta_{p}\right)$ ). The degrees of these extensions over $\mathbb{Q}$ are $p(p-1)$ and $p q, q \mid p-1$, respectively. The structure of their Galois groups is well known (see [4, p. 163]). The intersection of these Galois extensions is a subfield of $\mathbb{Q}\left(\zeta_{p}\right)$.

\section{References}

[1] A. Borodin, R. Fagin, J. Hopcroft, M. Tompa, Decreasing the nesting depth of expressions involving square roots, J. Symb. Comput. 1 (1985), 169-188.

[2] R. L. Borger, On DeMoivre's quintic, Amer. Math. Monthly 15 (1908), 171-174.

[3] Th. L. Heath (ed.), The Thirteen Books of Euclid's Elements, vol. III, Dover, New York, 1956.

[4] B. Huppert, Endliche Gruppen I, Springer, Berlin, 1979.

[5] G. Horng, M. Huang, On simplifying nested radicals and solving polynomials by pure nested radicals of mimimum depth, Proceedings of the 31st Annual IFEE Symposium of Foundations of Computer Science, 1990, 847-854.

[6] K. Ireland, M. Rosen, A Classical Introduction to Modern Number Theory, Springer, New York, 1982.

[7] W. Koepf, Hypergeometric Summation, Vieweg, Braunschweig, 1998.

[8] S. Landau, A note on Zippel denesting, J. Symb. Comp. 13 (1992), 41-45.

[9] S. Landau, How to tangle with a nested radical, Math. Intelligencer 16 (1994), 49-55.

[10] S. Lang, Algebra, Addison-Wesley, Reading (Mass.), 1971.

[11] B. K. Spearman, K. S. Williams, DeMoivre's quintic and a theorem of Galois, Far East J. Math. Sci. 1 (1999), 137-143.

[12] R. Zippel, Simplification of expressions involving radicals, J. Symb. Comp. 1 (1985), 189-210.

Kurt Girstmair

Institut für Mathematik

Universität Innsbruck

Technikerstr. 13/7

A-6020 Innsbruck, Austria

Kurt.Girstmair@uibk.ac.at 